\title{
GMT M1 subsystem: status, design and testing
}

Francisco Aguayo, Dave Ashby, Erich Bugueno, Barbara Fischer, John Ford, et al.

Francisco Aguayo, Dave Ashby, Erich Bugueno, Barbara Fischer, John Ford, Peter Gray, Michael Gusick, Anthony Hebert, Zaven Kechichian, Trupti Ranka, Dev Sharma, Jose Soto, Hector Swett, Divya Thanasekaran, Frank Tian, Abhijit Wadhavkar, "GMT M1 subsystem: status, design and testing," Proc. SPIE 10700, Ground-based and Airborne Telescopes VII, 1070036 (6 July 2018); doi: 10.1117/12.2312937

Event: SPIE Astronomical Telescopes + Instrumentation, 2018, Austin, Texas, United States 


\title{
GMT M1 Subsystem: Design, Status, and Testing
}

\author{
Francisco Aguayo*1, Dave Ashby ${ }^{1}$, Erich Bugueno ${ }^{1}$, Barbara Fischer ${ }^{1}$, John Ford ${ }^{2}$, Peter Gray ${ }^{1}$, \\ Michael Gusick ${ }^{2}$, Anthony Hebert ${ }^{1}$, Zaven Kechichian ${ }^{1}$, Trupti Ranka ${ }^{1}$, Dev Sharma ${ }^{1}$, Jose Soto ${ }^{1}$, \\ Hector Swett ${ }^{1}$, Divya Thanasekaran ${ }^{1}$, Frank Tian ${ }^{1}$, Abhijit Wadhavkar ${ }^{1}$ \\ ${ }^{1}$ Giant Magellan Telescope Organization, 465 N Halstead Street, Suite 250, Pasadena, CA 91107; \\ ${ }^{2}$ University of Arizona, Tucson, AZ 85721
}

\begin{abstract}
This paper describes the design, status, and test program for the Giant Magellan Telescope (GMT) Primary Mirror Subsystem (M1). It consists of the mirror cells, positioning system, support systems, and thermal control system. The seven $8.4 \mathrm{~m}$ mirror segments are excluded from this paper because they are considered a separate subsystem of the M1 System.

The M1 Subsystem leverages heritage design of similar telescope systems; for example, the Magellan telescopes and the Large Binocular Telescope. The M1 Subsystem incorporates pneumatic force actuators, hardpoints, and a thermal control ventilation system.

Design developments have been introduced to address the challenging levels of performance and unique requirements needed by the GMT telescope. Imaging goals necessitate an increase in mirror support performance, figure control, and higher-levels of thermal control. Additionally, there are challenges associated with matching and tracking the relative position of the seven mirror segments for mirror phasing. The design of the static support system needs to protect the mirrors from loads transmitted through the structure during an earthquake. Finally, the telescope design with interchangeable off-axis mirror cells necessitate mirror cells and support components that function under any range of gravitational vector orientations.

A full-scale Test Cell prototype is being constructed including production versions of mirror cell components to test and validate the M1 subsystem design. A Mirror Simulator will be used with the Test Cell to validate the M1 Control System. Later, a primary mirror segment will be used with the Test Cell to perform optical tests at the University of Arizona.
\end{abstract}

Keywords: Active Optics, Active Support System, Actuator Calibration System, Hardpoint, Heat Exchanger, GMT, M1, Primary Mirror, Static Support, Support Actuator, Test Cell

\section{INTRODUCTION}

The M1 Subsystem controls the position, surface figure, and temperature of the seven primary mirror segments. The position of each mirror is controlled by a hexapod design composed of six Hardpoints. The Hardpoints provide 6 degrees of freedom (DoF) and active alignment between a primary mirror segment and the corresponding secondary mirror segment. The surface figure of a mirror is actively controlled by pneumatic Support Actuators that are attached to the back surface of a mirror. The Support Actuators apply axial and lateral loads to correct for distortions in the glass caused by gravity sag in the mirrors. Together the Hardpoints and Support Actuators form what is referred to as an Active Support system. The temperature of the mirror is controlled by actively ventilating the internal honeycomb core and back surface of each mirror. The thermal control system consists of heat exchangers attached to fan assemblies that force conditioned air through the system. Additionally, Static Supports are used to support the weight of the mirrors when the Active Supports are not in use and protects the mirrors by absorbing energy and limiting their motion during a seismic event.

\footnotetext{
${ }^{1}$ faguayo@gmto.org; Phone +1 6262040500 x5236; http://www.gmto.org
} 


\subsection{Design}

\section{HARDPOINT}

The M1 hardpoints are part of a 6-DoF rigid body positioning system that are used for maintaining position and controlling M1 optical alignment between mirror segments. They are mounted to stiff points on the cell weldment and are attached to interface plates (wedges) that are bonded to the back surface of the mirrors (Figure 1).
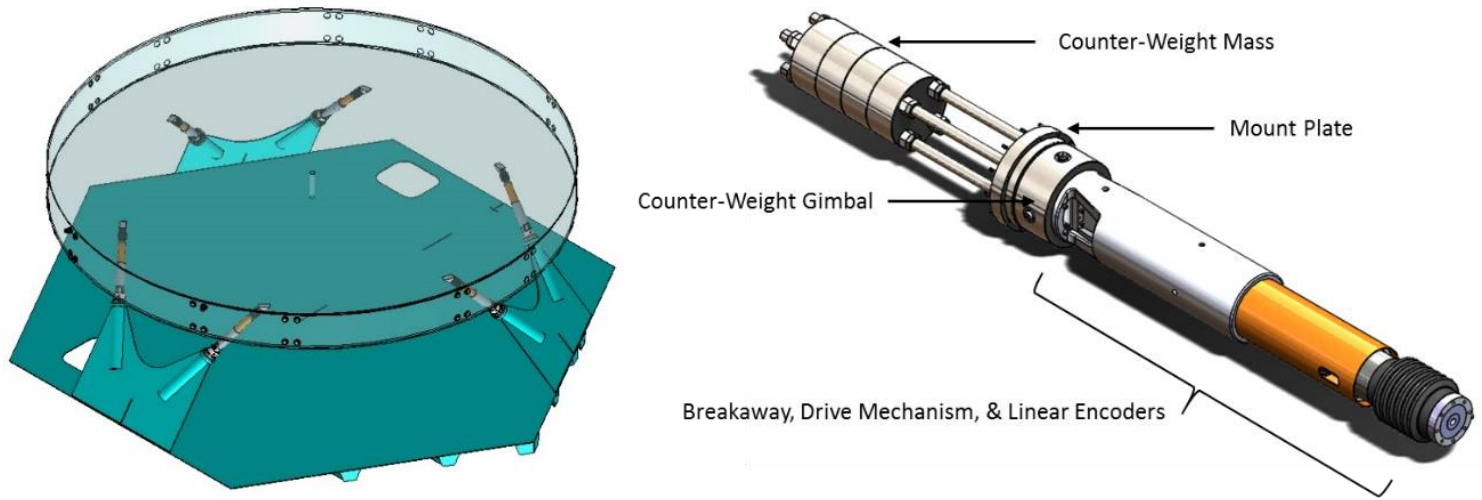

Figure 1: Hardpoint configuration on an Off-Axis Mirror Cell

The mirror can be positioned by varying the length of the hardpoints. The lateral forces and moments at the mirror interface points are minimized by the design of the hardpoint. During normal operations, there is ideally little to no force on the mirror at the hardpoint interface locations. Since the hardpoints are the only solid attachment between the mirror and the mirror cell, unacceptably high forces at the interface points are possible during a seismic event or Active Support system malfunction. A breakaway mechanism is designed in series with the hardpoint to limit the axial forces being applied to the mirror.

\subsection{Status}

Several design changes are currently being worked for the hardpoints. One such change includes increasing the stroke length of the hardpoint from $\pm 16.5 \mathrm{~mm}$ to $\pm 18.5 \mathrm{~mm}$. Additionally, the breakaway travel has been revised from \pm 45.5 $\mathrm{mm}$ to $\pm 60.0 \mathrm{~mm}$. These changes were made to accommodate revised motion budget allocations. However, the interfaces to the cell structure and the mirror were kept the same and the overall design envelope of the hardpoint remains unchanged.

DC Motors and Strain Wave Gears for the development Hardpoint Assemblies are currently on order and are expected to arrive in time for assembly of the hardpoints and integration of them with the Test Cell. Other long lead items, such as the roller screws and machined components, will begin their procurement and production process within the next month.

\subsection{Testing}

Testing of a prototype hardpoint has been ongoing for the last several months. Earlier tests revealed that the prototype did not meet its axial stiffness requirement $(\geq 120 \mathrm{~N} / \mu \mathrm{m})$ and that it was not repeatable. The hardpoint was disassembled and inspected. It was revealed that the surface roughness of contact surfaces was machine-rough. Those surfaces were remachined to provide better contact and the hardpoint was then reassembled. Subsequent testing showed that the hardpoint now meets its stiffness requirement and is repeatable. Additionally, the hardpoint test stand was modified to include an interferometer which provides better resolution in measuring the stiffness of the hardpoint and higher precision in measuring its travelled motion.

\section{SUPPORT ACTUATOR}

\subsection{Design}

Support Actuators are pneumatic force actuators that provide distributed support to the mirror cell segments. They apply a controlled force vector to a load spreader which then distributes the load to the back surface of the mirrors. The actuators 
correct for deformations that are created by gravity sag in the mirror, which varies with the elevation angle of the telescope. They also correct for mirror surface figure errors as commanded by the Active Optics (AcO) control loop. The actuators come in two types: single-axis and triple-axis actuators (Figure 2). Single-axis actuators can only apply a force in the axial direction normal to the back surface of a mirror. Triple-axis actuators can apply forces in 3 directions: one normal to the back surface and two in-plane of the back surface of the mirror. Actuators are mounted on the top plate of the mirror cell and interface with load-spreaders that are permanently bonded to the back surface of the mirror.

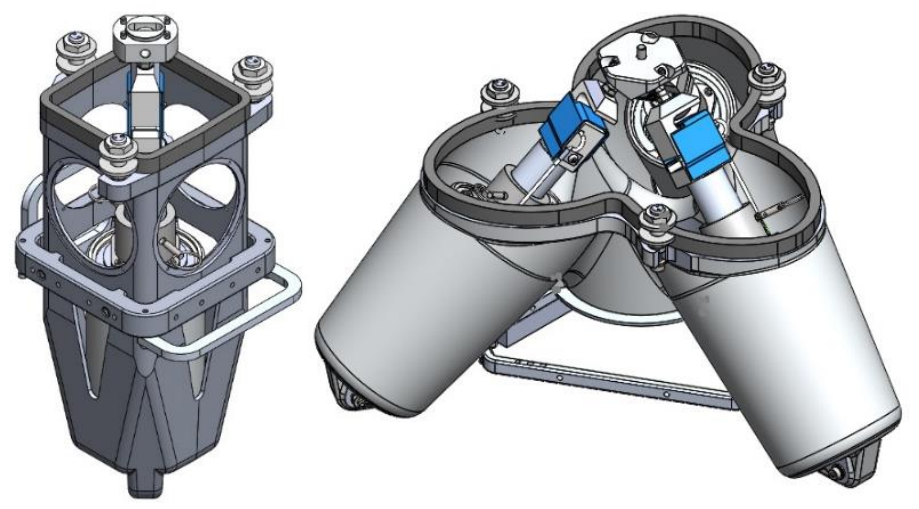

Figure 2: Single and Triple-Axis Support Actuators

The portion of the support actuator that applies the force is called the Air Cylinder assembly. The Air Cylinder assembly is identical for both the single and triple-axis actuators. The single-axis actuators have one air cylinder and the triple-axis actuators have three air cylinders (Table 1). The Air Cylinder assembly houses a rolling diaphragm that when pressurized applies a force onto the piston which transmits the force through the shaft to the attached load cell and load spreader interface plate. Each Air Cylinder assembly has a total stroke length of $\pm 40 \mathrm{~mm}$. At each end of the Air Cylinder assembly, there are rod ends which allow for 3 degrees of rotation. The rod ends eliminate most of the moment at the "working point" where a force vector is applied on a load spreader.

Table 1: Air Cylinder Assemblies for Single and Triple-Axis Actuators

\begin{tabular}{|c|c|c|c|}
\hline Cell Type & $\begin{array}{c}\text { Single-Axis } \\
\text { Actuators }\end{array}$ & $\begin{array}{c}\text { Triple-Axis } \\
\text { Actuators }\end{array}$ & $\begin{array}{c}\text { Air Cylinder } \\
\text { Assemblies }\end{array}$ \\
\hline Off-Axis & 80 & 90 & 350 \\
\hline On-Axis & 78 & 76 & 306 \\
\hline
\end{tabular}

The air pressure inside of the Air Cylinder assembly is controlled by the Valve Manifold assembly. The Valve Manifold assembly controls the amount of air pressure that is applied to either side of the piston, thereby controlling the force that is applied to a load spreader. The assembly is identical for both types of actuators. The Valve Manifolds are mounted to the exterior of the support actuator housing in different locations for the single and triple-axis types. These assemblies were previously only intended to house the air valves but now include the remaining components (safety solenoid valves, check valves, and air filters) that it needs to make each Support Actuator a line replaceable unit.

The Support Actuator Controller communicates with the cell software and forms what is part of the AcO control loop. The Controller consists of a Printed Circuit Board (PCB) and the controller firmware. The PCB is physically identical for both the single and triple-axis actuators. However, the mounting locations on the actuator assembly are different. For the singleaxis actuator, it is mounted in one of the four large hole cut-out regions near the top of the actuator housing. For the tripleaxis actuator, it is mounted below the actuator housing. The Controller identifies itself to the Cell Software. 


\subsection{Status}

Components for the development of the Support Actuator assembly are currently being fabricated. Custom parts and COTS components are on order. The development units will be used to support system level testing on a Test Cell assembly which simulates an M1 off-axis cell. Aluminum castings of the actuator housings for both types of actuators are in fabrication.

A damper design is being explored to be included in the Air Cylinder Assembly. The damper would provide viscous damping to the piston motion. This dampens large piston motion in hazardous situations such as an earthquake. Without them the Support Actuators would bleed out air during an earthquake and the mirrors would be lowered down onto the Static Supports. This design is currently in its prototype phase after which its performance will be evaluated.

\subsection{Testing}

Component and unit level testing of the support actuators will take place prior to the integration of the development units onto the Test Cell assembly.

Rod ends will be tested to characterize the amount of friction inside of the bearings. A sample lot will be tested at ambient temperature to measure their coefficient of friction. Rod ends to be used on the development assemblies will be selected based on a statistical analysis of the lot.

Every Air Cylinder assembly will be pressurized and leak checked before they are integrated with the rest of the support actuator assembly. A dedicated stand has been developed to perform this activity as hundreds of actuator assemblies need to be verified prior to installation.

Prior to integrating a Support Actuator onto a cell, each unit will undergo a series of qualification and calibration tests. A dedicated test stand, the Actuator Calibration System (ACS), is currently being development and will be used to test the first set of development units being integrated onto the Test Cell. The ACS will first calibrate each Support Actuator to achieve repeatable and precise force setting accuracy. The ACS will then qualify a Support Actuator for use after passing the qualification acceptance criteria. If a Support Actuator fails, the ACS will disable the unit making it unusable in the mirror cell. The Support Actuator will only be operable again when it is repaired and calibrated.

\section{STATIC SUPPORT}

\subsection{Design}

Static Supports are passive spring isolators that support the weight of the mirrors when the telescope is not in use and protects the mirror during unexpected accelerations (i.e. during handling or a seismic event). The weight of each mirror is distributed among hundreds of Static Supports to keep the stresses in the mirror below their safe limits (Figure 3).
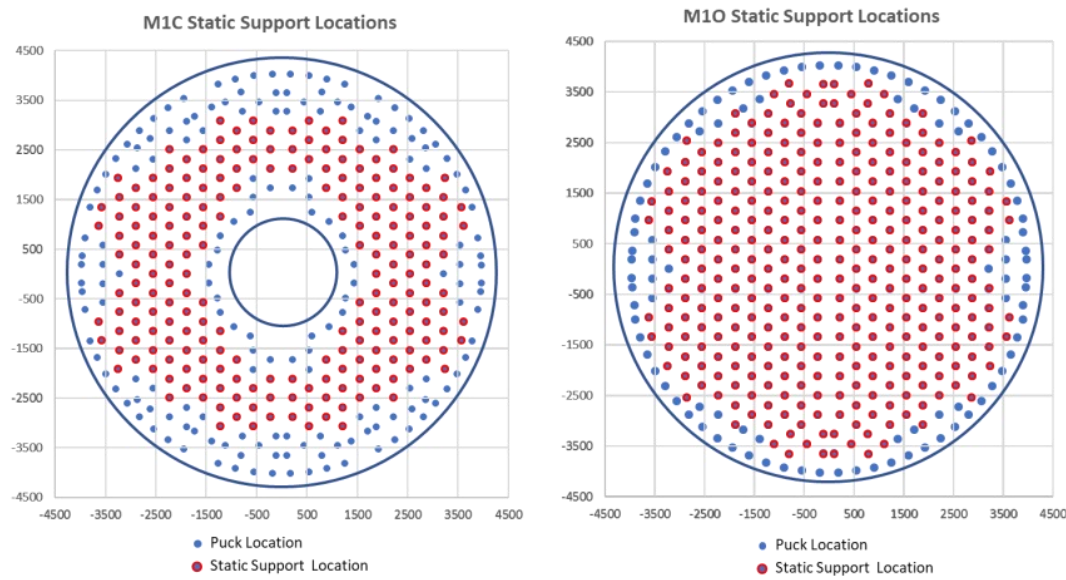

Figure 3: Locations of Static Supports 
The Static Support design is based off a modified commercial shock isolator consisting of wire rope arches secured in a basket shape between two plates (Figure 4). Each off-axis segment has 332 static supports and the center segment has 212. They interface with the top plate of the cell and the flanges of the load spreaders which are attached to the mirrors.

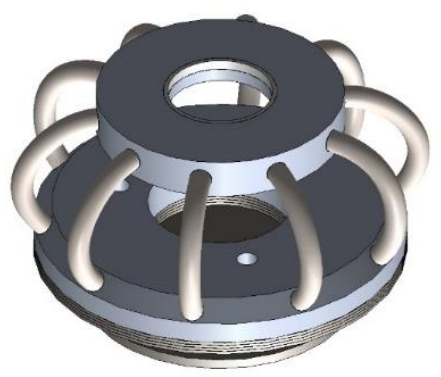

Figure 4: Static Support

\subsection{Status}

The design of the static supports is driven by environmental requirements, interface constraints, performance requirements, and dynamic requirements. Extensive analysis and testing has already been performed to converge on a baseline design for them. The Static Supports are currently undergoing an iterative design process as the dynamic requirements are currently being refined. These requirements are driven by the consideration of seismic accelerations that may be experienced at the site, which are applied to an integrated model to analyze the motion of the mirrors. The result of this analysis will determine the displacement and stiffness requirements of the static supports. They will also determine if the baseline design can be matured or if a redesign of the static supports is necessary. Additionally, alternative designs are currently being explored as a fall back plan just in case the analysis determines that the baseline design is unviable.

\subsection{Testing}

GMTO will conduct in house prototype testing to ensure the static support design meets all environmental, performance and dynamic requirements. In addition, GMTO will also perform functional tests on each static support wire basket to determine its stiffness, deflections, and damping properties. The data collected will be used to sort the static supports and determine their locations on a mirror cell so that they meet installation and load distribution requirements.

\section{THERMAL CONTROL SYSTEM}

\subsection{Design}

The M1 Thermal Control System is designed to actively ventilate the primary mirror segments when the telescope is in use. The system maintains the temperature of the mirrors near or at the ambient temperature inside of the enclosure. The Thermal Control System is required to cool the mirrors so that the telescope can meet its image quality requirements as detailed in the GMT Science Objectives.

Thermometry is key to the thermal control design. Temperature sensors will be installed inside of the mirror cores and on their back surfaces. Additionally, sensors will be placed inside the lower plenum of the cells and on the fan units that circulate the air inside the plenum. The system will read the temperature of the ambient environment inside of the enclosure and then actively control the temperature of the primary mirrors to reduce the thermal gradient between the mirrors and their surrounding environment.

The M1 Subsystem makes use of a forced convection design for controlling the temperature of the primary mirrors. Each cell will be equipped with Fan Assemblies and two Radiators mounted on opposites sides of the Fan Assembly (Figure 5). The Radiators within a cell are connected in series by a network of plumbing lines and a dedicated pump for each cell circulates coolant throughout the network. The fan assemblies blow air across the radiators and moves the air from the lower plenum of the cell into the upper plenum and then onto the back surface of the mirror. Two types of air nozzles move air from the lower plenum into the upper plenum: Mirror Nozzles and Upper Plenum Nozzles. Conversely, a duct moves air from the upper plenum back down into the fan assemblies which forms a circulating loop. Air from the outside 
of the cell can mix with the air inside of it, making the system a semi-closed loop system. The Fan Assembly will come equipped with an accelerometer to monitor vibration levels during operation and identify if a fault in the fan has occurred.

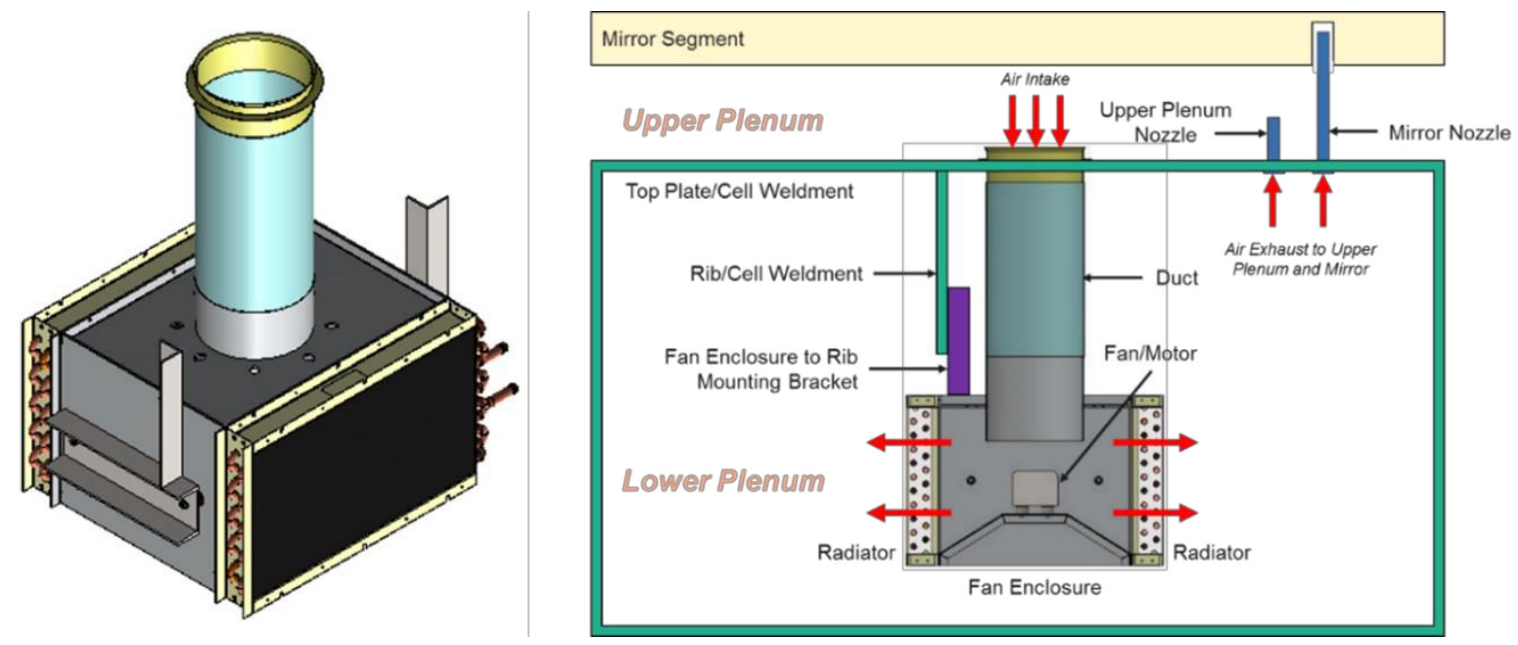

Figure 5: M1 Thermal Control System

\subsection{Status}

The Thermal Control System has had little change to its design since PDR. Several design activities, including thermal analysis, are under way to determine how many fan assembly units are required for each cell. Similarly, the analysis will determine if the upper plenum nozzles are required to improve mirror ventilation.

The design needs to be finalized by September 2018 to allow enough time for procurement and fabrication of components in support of system level testing with the Test Cell.

\subsection{Testing}

One of the design considerations for selecting a fan unit is the amount of vibration it induces into the cell. Testing of the fan unit will need to be performed to determine if its vibration signature is within the allowed requirement of the mirror cell and the mirror optical performance.

\section{UTILITIES}

\subsection{Design}

The M1 Subsystem requires several utilities to be supplied to each cell. Those include an Air Cabinet, a Power and Communication Cabinet, Utilities Distribution, a Bulkhead Panel, Service Lighting, and a Service Communication system. The Air Cabinet routes dry clean air to each cell where it is distributed to the Active Supports. The Power and Communications Cabinet provides power to the cell and communicates with the electrical components within the cell (for Active Supports and Thermal Control System). The Utilities Distribution system distributes utilities including air and coolant from outside of the cells into the lower plenums. The Bulkhead Panel serves as the interface between the cell electronic cabinets and a remote Cell Controller Cabinet which is housed external to the M1 System.

\subsection{Status}

The Utilities design is currently in work and details of the electronics cabinets such as the size, location within a cell, and cable routing are set to be finalized by September 2018. Space allocations within the M1 cells have been reserved for the electronics cabinets. The Mount team owns the design of the cell's steel structure and interfaces between the two are being coordinated. 


\subsection{Testing}

The Utilities will be checked out and functionally tested at the system level test with the Test Cell. This test will include development units of all the M1 hardware and will have all the interconnectivity and control system of the production cells. This test is currently planned for the early part of 2020.

\section{CELL ELECTRONICS}

\subsection{Design}

The Cell Electronics is a distributed control system based on the EtherCAT field bus. The Cell Electronics are designed to control the figure and position of the mirror, protect the mirror from damage in the event of a component failure, or if an external influence such as air, moisture, or coolant is out of specified ranges. It is controlled by the M1 Control Computer (MCC), an industrial computer that serves as the local EtherCAT master. An EtherCAT connection monitors and controls the DC power supplies that power the cell. The DC supplies are redundant, allowing operation even with a faulted $48 \mathrm{~V}$ or $24 \mathrm{~V}$ power supply. Redundant DC buses provide power to the equipment inside the cell. Vibration sensors attached to the mirror cell are read via the EtherCAT connection from modules in the Cell Electronics Cabinet. Readout and processing of the mirror temperature sensors is accomplished via an Ethernet-connected thermal sensing system. The MCC provides a processed set of mirror temperatures to the thermal control and mirror safety system. Support Actuators within a cell have an embedded control system attached to them which controls the pressure applied to the mirror. The Hardpoints have a control node with an intelligent motor drive, a length measurement module, and load cell measurement module. The Thermal Control System fans also have a control board attached to them. These devices are all connected via EtherCAT to the MCC.

\subsection{Status}

The Cell Control Electronics design is currently in work. Block diagrams of their interconnectivity are complete and component selection is nearly complete. Detailed electrical design and drafting is set to complete by September 2018.

\subsection{Testing}

Cell electronics will be tested at the system level with all other M1 hardware integrated with the Test Cell.

\section{INTERLOCK AND SAFETY SYSTEM}

\subsection{Design}

The M1 Interlock and Safety System (ISS) is designed to safeguard the mirror from damage in the event of an external or internal failure of control system or utilities. The two main fault modes for damaging a mirror are thermal shock and excessive force application. Other fault modes are mitigated by adding redundancy in things such as telemetry sensors and power supplies, and air regulators on the support actuators. The M1 ISS monitors the air supply quality, power systems, and thermal system control.

The M1 ISS consists of a Beckhoff TwinCAT Programable Logic Controller (PLC) and associated analog and digital I/O modules. The PLC controls the air supply into the cells and the temperature of the mirror. The air supply to the mirror is monitored to ensure that the control valves operate nominally. A differential pressure sensor monitors the pressure drop across the incoming filter. Then, a flow meter monitors the flow through the system to detect excessive flows. Additionally, dew point sensors are used to assess the air quality. Pressure sensors indicate the pressure in the manifold of each air system. Together, these sensors are used to ensure that the mirror cell is supplied clean air of the correct pressure. If any of these sensors indicate a situation that could lead to damage of a mirror or the cell equipment, the M1 ISS will command the air valves to close, thereby lowering the mirror back onto the Static Supports. A signal from the global ISS can also command the mirror to be lowered. A hardware watchdog timer has been incorporated to detect if the M1 ISS has failed. If the watchdog detects that the ISS has failed then it can command that the mirror be lowered without any intervention from any other devices. The thermal control portion of the M1 ISS monitors and controls the temperature that is supplied to the mirror cell. Redundant pumps provide coolant to the network of heat exchangers inside of a cell. 
Vibration sensors and Bearing Failure Detectors are mounted on each pump to assess its health. Redundant thermal sensors also monitor the coolant temperature to ensure that the coolant entering the cell is at set to the right temperature. A dew point sensor inside the cell is monitored to ensure that the cell temperature does not fall below the dew point. This protects the cell from the harmful effects of condensation.

\subsection{Status}

ISS block diagrams have been developed and component selection is underway. This work is set to complete before the end of November 2018.

\subsection{Testing}

The ISS will be tested with the rest of the M1 Subsystem as part of the system level testing with the Test Cell.

\section{DEVICE CONTROL SOFTWARE}

\subsection{Design}

M1 Device Control Software (M1 DCS) will be responsible for implementing the real-time control system of the Test Cell. The main variables controlled by the M1DCS are 6 DoF position of the segment, the active support forces applied to the mirror, and the temperature of the glass segment. The real-time communication network inside the cell is EtherCAT. GMT has adopted an architecture where the M1 cell software running in the M1DCS PC is an EtherCAT. The low-level devices controlling each of the test cell components are EtherCAT slaves. Figure 6 shows the M1DCS master and slaves within the Test Cell. The EtherCAT slaves will have COTS and custom-built components. The custom-built hardware will have the same embedded platform and base firmware which will be modified as required by the subcomponents. 


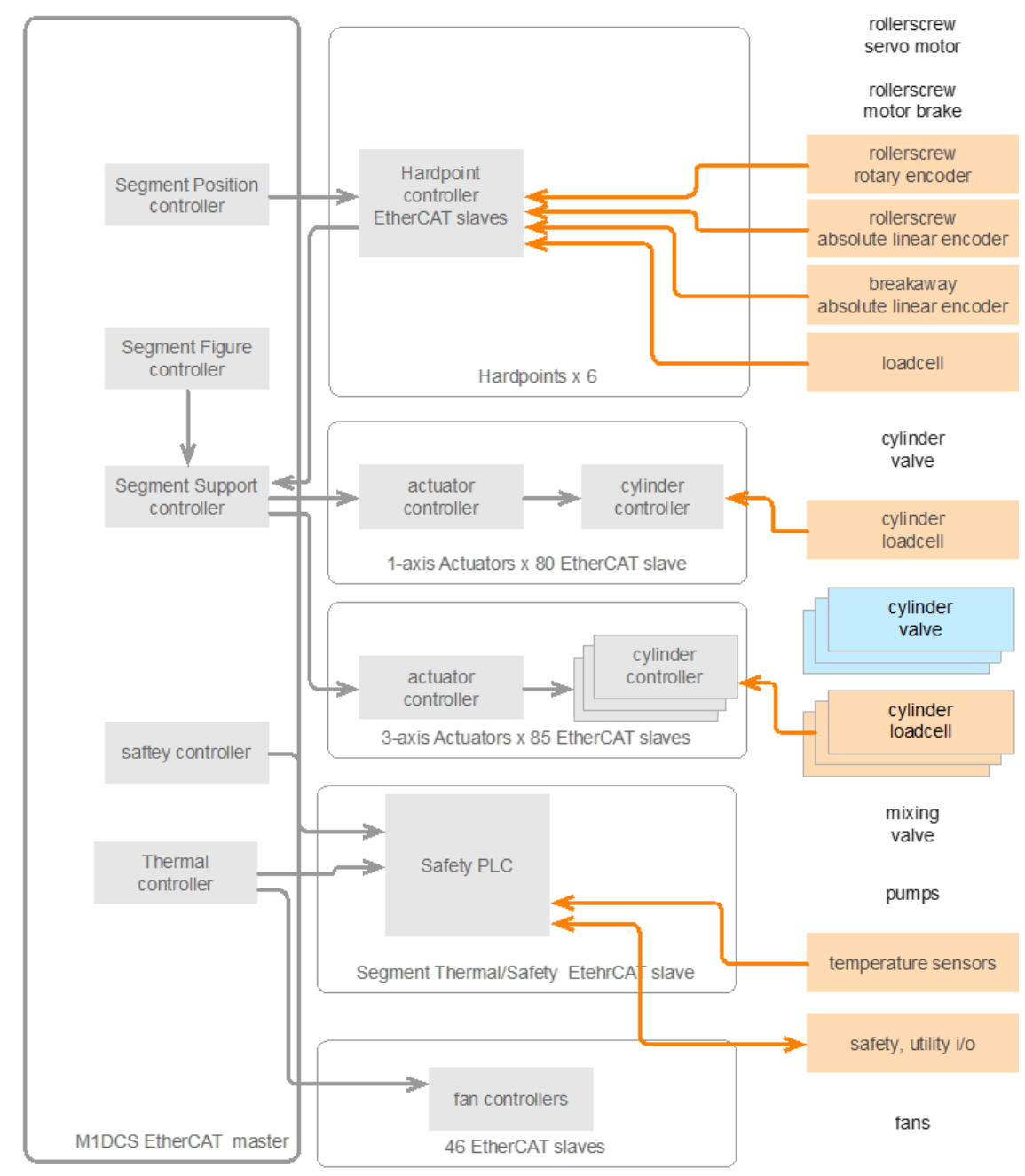

Figure 6: M1DCS EtherCAT Master and Slaves

The M1DCS software will be modified so that it can be implemented in GMTO software framework and run on real-time RT_PREEMPT Linux OS. The system will be defined using a model based system definition as has been adopted by the GMTO Software Team. Figure 7 shows the model description implemented in coffee script.

\subsection{Status}

The M1DCS software is in requirements development and a design phase. Its implementation is expected to begin in August 2018. 


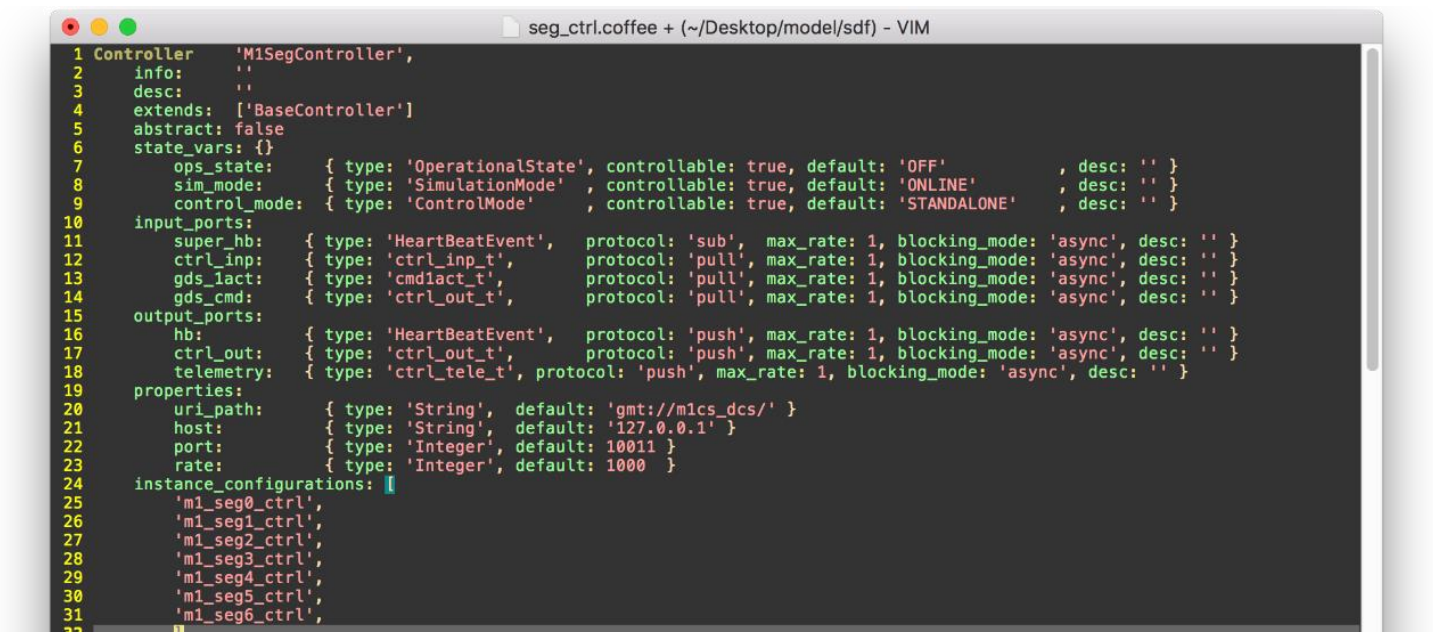

Figure 7: M1DCS System Description in CoffeeScript

\subsection{Testing}

The M1 custom EtherCAT slaves are embedded systems with 32-bit microcontrollers to run the slave application. The slave for support actuators have been prototyped and are now in their test phase. Figure 8 shows the testing of a prototype slave. This slave will be modified to implement fan controllers and power and communication hubs.

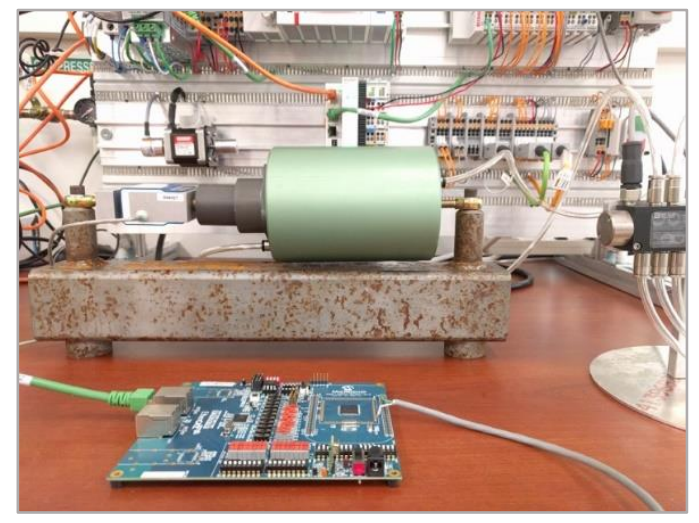

Figure 8: Support Actuator Custom EtherCAT Testing with Actuator Hardware

\section{ASSEMBLY, INTEGRATION, AND TESTING}

The M1 Subsystem's performance relies on the proper assembly and integration of the GMT mirror cells. Installation and accurate alignment of support actuators and hardpoints, within the confined space of a cell, poses several challenges. The accurate placement of actuators relative to the mechanical interfaces of the mirror segment load spreaders is critical to ensure the performance and safety of the mirrors. Hardpoints must be installed at precise locations and mounted correctly to ensure they work as intended. Alignment of the Static Supports with the underside of the mirror segment is essential to ensure glass safety and mirror support performance. Additionally, a large amount of utility plumbing is needed in the mirror cell to supply air to the pneumatic actuators and coolant to the thermal control units. Power and network cabling will need to be distributed within the cell and electronics modules will need to be mounted to a thermally controlled enclosure.

A Test Cell Weldment is currently being fabricated to help validate the M1 Subsystem design and debug issues prior to final cell integration with production hardware (Figure 9). This full-scale "flight-like" version of an off-axis GMT mirror cell will first be used to fit check the M1 Subsystem components and verify their mechanical interfaces. Next, a steel M1 Simulator will be used to validate the M1 support system. Finally, a GMT glass mirror segment will be integrated with the 
Test Cell and the M1 Subsystem will be tested for its optical performance using the University of Arizona's Optical Test Tower located in the Richard F. Caris Mirror Lab.

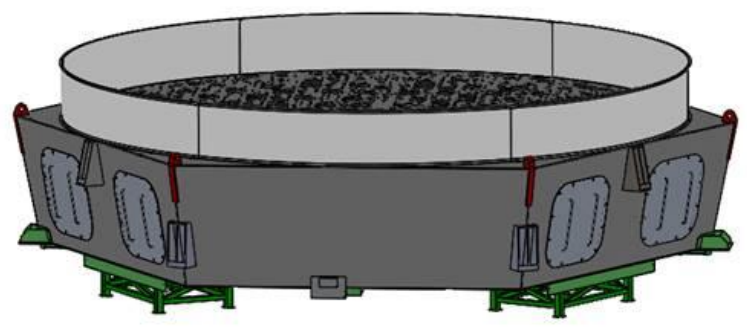

Figure 9: M1 Test Cell Weldment

The M1 Simulator is a test apparatus used for validating the primary mirrors control system. A Request for Proposal (RFP) is currently being developed which will identify a suitable contractor to build the M1 Simulator. The M1 Simulator will be fabricated from steel material and will have similar mass properties and interface features as the primary mirrors (Figure 10). This allows for safe testing and validation of the M1 Subsystem with reduced risk to the glass mirror segments. Finetuning of mass properties is made possible by using the simulator's integrated mass calibration system. The mass calibration system is used to configure it for either on-axis and off-axis mirror simulator.

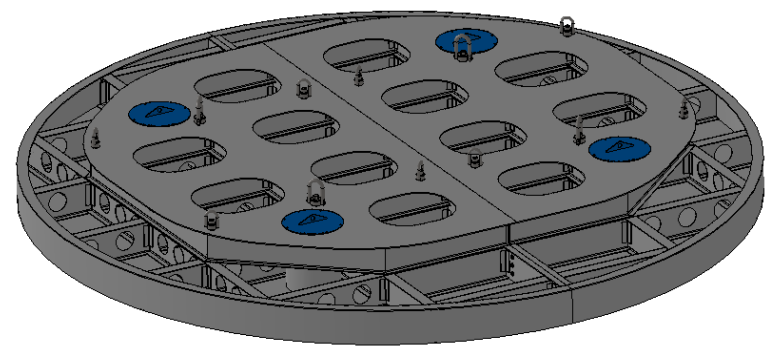

Figure 10: M1 Mirror Simulator

The integration phase has the additional challenge of having schedule constraints as this will drive the date of "first light" of the telescope. Assembly and integration work of M1 hardware on the cell structure can start once the cell structure has been delivered by the vendor. That work can be performed in the U.S. prior to it being shipped to the site in Chile. After the hardware has been delivered to the site, the cells can then be installed and fit checked with the telescope structure. Next, the cell will be removed from the telescope structure and final assembly and integration of all other remaining M1 hardware can continue. The last piece to be integrated with a cell will be the glass mirror segment. This process will be repeated 6 additional times for all remaining GMT M1 cells.

\section{SUMMARY}

The M1 Subsystem team has been working diligently to finalize the requirements and design in preparation for fabrication of development hardware and system level testing with a Test Cell. The design of the Active Supports has previously been given priority. However, recent personnel additions to the M1 team have allowed for further progress in the development of other hardware. The design of the Utilities, Static Supports, and the Thermal Control System are planned to be finalized before the end of 2018 to allow sufficient time to procure parts for the development of those assemblies. The Test Cell weldment is currently in fabrication and is scheduled for completion by start of 2019. The M1 Simulator is currently in procurement phase and fabrication is due to begin by the end of 2018. Component and unit level testing will be performed prior to integration of the hardware within the Test Cell. This will help identify and correct issues of the M1 design before those components are assembled and tested as a system. Achieving these milestones will allow GMT testing of the M1 Subsystems to commence by the early part of 2020 . 


\section{ACKNOWLEDGEMENTS}

This work has been supported by the GMTO Corporation, a non-profit organization operated on behalf of an international consortium of universities and institutions: Arizona State University, Astronomy Australia Ltd, the Australian National University, the Carnegie Institution for Science, Harvard University, the Korea Astronomy and Space Science Institute, the São Paulo Research Foundation, the Smithsonian Institution, the University of Texas at Austin, Texas A\&M University, the University of Arizona, and the University of Chicago.

\section{REFERENCES}

None 